\title{
L'état des études françaises, francophones et globales dans les universités américaines
}

\author{
Véronique Maisier \\ Southern Illinois University Carbondale
}

\section{1) Introduction}

En 1998, je débutais à mon poste universitaire actuel en tant que professeure assistante. À l'époque, nous étions quatre professeurs titularisés ou titularisables dans la section de français, répartis selon les spécialisations traditionnelles en littérature française du XVIII ${ }^{\mathrm{e}}$ siècle, en littérature française du $\mathrm{XIX}^{\mathrm{e}}$ siècle, en pédagogie et linguistique, plus ma propre spécialisation en littérature française du $\mathrm{XX}^{\mathrm{e}}$ siècle. Qu'en est-il aujourd'hui? Je suis la seule professeure titularisée dans cette section et nous engageons chaque année un lecteur ou une lectrice de français (c'est-à-dire un professeur non titularisable avec un contrat déterminé et renouvelable selon la disponibilité des budgets) pour m'assister dans l'enseignement des classes requises pour la spécialisation de français. L'évolution de notre section de français, dans un département de langues et littératures étrangères - bientôt rebaptisé "langues, cultures et commerce international" - dans une université d'environ 20000 étudiants offre un excellent point de départ pour une discussion sur l'état des études françaises, sur le statut de la langue française et sur ses relations avec les études francophones et les études globales.

\section{2) L'enseignement de la langue française}

Il importe dans un premier temps de différencier le terme "français » en tant que langue enseignée aux États-Unis et «français » en tant que discipline ou domaine d'étude. Malgré les nouvelles fréquentes ces dernières années de fermetures de départements de français dans diverses universités américaines et malgré mon expérience personnelle d'érosion du programme de français à Southern Illinois University à Carbondale, le français en tant que langue enseignée aux États-Unis se porte généralement plutôt bien. Quelques statistiques appuient cet état de fait, comme par exemple le rapport «Enrollments in languages Other than English in U.S. Institutions of Higher Education » publié par Nelly Furman, David Goldberg et Natalia Lusin sur le site web de la Modern Language Association (MLA), daté de 2010, qui rapporte les résultats d'un sondage de 2009. Selon les statistiques données dans ce rapport, le français s'impose comme deuxième langue la plus étudiée dans les universités américaines. Le taux de croissance de son enseignement de 
$4,8 \%$ est tout à fait encourageant comparé aux autres langues modernes européennes qui présentent un taux de croissance de 5,1\% pour l'espagnol, $2,2 \%$ pour l'allemand, 3\% pour l'italien. Ces taux de croissance sont certes plus élevés en ce qui concerne les langues dites cruciales - ou "critical languages " - avec 18,2\% pour le chinois, $19,1 \%$ pour le coréen, $46,3 \%$ pour l'arabe. Remarquons également la forte augmentation des classes de American Sign Language (ASL) dont le taux de croissance de 16,4\% place cette langue e deuxième position avant le français dans les two year colleges ${ }^{2}$. En nombre absolu d'étudiants, le français se place après l'espagnol qui avec 864986 étudiants regroupe plus d'étudiants que toutes les autres langues modernes réunies (764 340 étudiants) mais nettement avant les autres langues avec 216419 étudiants en français ${ }^{3}$ comparés à 96346 pour l'allemand en troisième position et 35083 pour l'arabe en huitième, par exemple. Les conclusions qui s'imposent à la lecture de ce rapport sont que le français en tant que langue enseignée aux États-Unis n'est pas immédiatement en crise, même si certains indices attestent de la nécessité de rester vigilants. Incidemment, l'étude de Louis-Jean Calvet sur la place du français dans le monde basée sur une analyse de dix facteurs explicités ci-dessous aboutit sensiblement à la même conclusion précautionneuse :

To summarize, we can see that French is amongst the highest ranking languages in a number of factors: number of countries in which it is an official language (second place), use by international radio stations (second place), Nobel Prizes for Literature (second place), source language in translation (second place), and number of Wikipedia articles (third place). The French language is lower-ranked in terms of its circulation across the internet (sixth place), and badly positioned in terms of the number of its speakers (nineteenth place). Its status as an international language (U.N.'s Secretariat and E.U.) is potentially under threat, as is its position as a foreign language being taught in secondary schools (148).

La situation est plus fluide encore pour ce qui est du français en tant que discipline qui a rencontré ces dernières années de profonds bouleversements manifestés dans la refonte de programmes ou l'addition de spécialisations -

1 Depuis le 11 septembre 2001, le gouvernement américain a développé un «language flagship» qui met l'accent sur l'enseignement des «langues cruciales » et qui fournit des avantages pour l'enseignement de ces langues. Ce programme, lancé en 2002, cherche à promouvoir ces langues afin de créer des «citoyens globaux» comme annoncé sur le site web à www.thelanguageflagship.org.

2 Nous suivons la distinction établie dans le rapport MLA entre two-year colleges et universités ou four-year institutions ciblés par le sondage : «In the end, the fall 2009 enrollments presented in this report are collected from a total of 2,514 AA-, BA-, MA-, and PhD-granting colleges and universities, representing $99.0 \%$ of all higher education institutions offering languages in the United States » (Furman et al. 1).

3 Ce nombre était plus élevé en 1960 (228 813), en 1968 (388 096), en 1980 (248 361), et en 1990 (272 472), mais plus bas dans les vingt dernières années: 205351 en 1995; 199064 en 1998; 201979 en 2002; et 206426 en 2006. (Voir Furman et al. 19-21). 
francophonie, pédagogie (ou Second Language Acquisition), cinéma et études culturelles parmi les plus fréquentes - afin de répondre à la demande des étudiants et du marché professionnel. Malgré les réformes plus ou moins considérables entreprises sur de nombreux campus américains, le français en tant que discipline éprouve toujours aujourd'hui des difficultés à se situer dans le contexte de l'émergence des études francophones et plus récemment des études globales ainsi que dans la mouvance de la crise des humanités de l'enseignement supérieur.

\section{3) La crise des études françaises traditionnelles}

Les études françaises traditionnelles avec leurs répartitions bien établies entre d'une part la linguistique, la littérature et la culture et d'autre part les séparations littéraires réparties par périodes entre le Moyen-Âge, le seizième siècle, le dix-septième, le dix-huitième, etc., connaissent un recul marqué ces dernières années comme l'atteste par exemple la liste des propositions d'emplois de la MLA de l'automne 2013. Les données disponibles ne nous habilitent pas à offrir ici de statistiques ou de sondage scientifique car la liste analysée reste relativement limitée et couvre uniquement l'année académique 2013-2014. Cette liste nous permet cependant de tirer quelques conclusions sur les tendances actuelles et de noter en particulier un grand intérêt pour les généralistes dans les descriptions de postes à pourvoir. Ainsi, la répartition traditionnelle par siècles spécifiques se trouve remplacée par des expressions comme «avant 1800 ou après 1800 » qui couvre des périodes plus grandes que par le passé tandis que l'on cherche par exemple un "généraliste avec expertise en $\mathrm{XIX}^{\mathrm{e}}$ et $\mathrm{XX}^{\mathrm{e}}$ siècle».

Lorsque la distinction par siècles est maintenue, elle s'adresse le plus souvent aux experts du vingtième et du vingt-et-unième siècle, regroupés dans une même catégorie. On remarque une tendance à l'agglutination par exemple dans des descriptions telles que «20th/21st century literature and film», «20th/21st century French/Francophone literatures » ou encore «French and Francophone Studies ». Les positions par siècle littéraire sont toutefois fréquemment remplacées par des spécialisations postmodernes telles qu'études médiatiques, relations culturelles transnationales, environnement et écocritique, par exemple. Comme le souligne Larry Norman en 2006 :

$\mathrm{Au}$ moment où l'on remet en cause les frontières nationales et linguistiques, l'essor des études culturelles entre autres tendances actuelles (gender, post-colonial, cinema et media studies, par exemple) - brouillent les anciennes démarcations disciplinaires et méthodologiques (21-22).

Si la terminologie et les dénominations importent moins que l'obtention d'un emploi pour les jeunes doctorants qui sortent de nos programmes, elles n'en 
restent pas moins révélatrices des changements qui se sont produits vis-à-vis des anciennes démarcations et de la réévaluation de la mission des professeurs de français. Les enjeux associés à cette mission aussi bien pour les études du français en tant que discipline que pour l'avenir des carrières académiques et du professorat et pour la qualité des programmes universitaires et le succès des étudiants sont vivement ressentis par les professeurs qui se voient souvent remplir aujourd'hui « en solo» les fonctions autrefois réparties entre plusieurs collègues.

Se trouve alors mise en relief plus que jamais l'importance de se montrer judicieux dans les propositions d'emploi afin de satisfaire aux besoins des programmes ainsi que pour faciliter la tâche de convaincre les administrateurs des avantages à engager un généraliste, expert en multiples domaines et, qui plus est, versé dans l'interdisciplinarité et apte à enseigner dans divers départements ${ }^{4}$. La demande de professeurs généralistes met l'accent sur l'embauche perçue comme un investissement dont on espère tirer le plus de profit possible et révèle le besoin réel d'une certaine flexibilité au niveau des classes enseignées dans le département ou entre plusieurs départements. On remarque de plus l'intérêt marqué pour des professeurs capables d'enseigner une langue autre que le français, et en particulier une langue «cruciale » comme l'arabe: sur les dix-neuf positions affichées sur la liste de la MLA le 10 décembre 2013 (MLA Job Listing), cinq exigeaient la connaissance d'une troisième langue en plus du français et de l'anglais à un niveau avancé, la troisième langue étant l'arabe, l'italien, le latin ${ }^{5}$ ou au choix.

Dans sa contribution au célèbre volume intitulé Pour une littérature-monde (2007), Alain Mabanckou établissait «le portrait-robot de l'écrivain en langue française confronté au monde» (Mabanckou 55). Qu'en est-il du portraitrobot du professeur de langue française confronté à ce même monde? Son existence et son espérance de vie diffèrent grandement selon qu'il opère dans le monde globalisant qui fait de lui un citoyen du monde dont le français risque de se trouver expulsé au profit de l'anglais, du mandarin, de l'arabe ou de l'hindi; dans le monde académique qui lui demande de poursuivre une recherche approfondie de spécialiste; dans le monde universitaire avec la réalité de ses coupures de budgets, des priorités des étudiants-consommateurs et des

\footnotetext{
${ }^{4}$ «Dans ce contexte, rien n'est plus naturel que de se demander si les anciennes disciplines ont encore un sens. Ce qui est sûr, c'est que les départements de français ont toujours intérêt à mettre en valeur les travaux interdisciplinaires au cours de leurs négociations avec l'administration centrale. Car les vieilles raisons d'être des études littéraires nationales ne convainquent plus guère » (Norman 30).

${ }^{5} \mathrm{Il}$ s'agit ici d'une position pour un médiévaliste devant enseigner $60 \%$ des classes en français et $40 \%$ des classes en latin.
} 
modes ou tendances disciplinaires; ou encore dans le monde du marché qui lui demande d'être généraliste, spécialiste, globalisant et prêt à enseigner en français, en anglais et de préférence dans une troisième langue. Ce professeur $\mathrm{du}$ monde postmoderne évoque paradoxalement l'bomo universalis de la Renaissance : que de chapeaux il lui faut porter, auxquels s'ajoutent dorénavant le chapeau francophone!

\section{4) L'impact des francophonies}

Les francophonies ${ }^{6}$ ont sans aucun doute un impact sur le français en tant que langue et système linguistique et en tant que discipline étudiée à l'université. Les études francophones dominent aujourd'hui nombre de départements français et imposent leur pertinence dans notre monde postcolonial qui paraît les mettre en compétition avec les études françaises. Antoine Compagnon remarquait en 2003 :

Le boom de la francophonie dans les études sur la littérature en français aux Etats-Unis est certes un phénomène nouveau, massif et remarquable, qui transforme en profondeur tous les départements de langue et littérature françaises, mais l'existentialisme, le structuralisme et le féminisme n'avaient pas eu des effets différents sur les générations précédentes. Pour tout doctorant, quelle que soit par ailleurs sa vocation, une certification complémentaire en francophonie (et en cinéma) est sans doute devenue indispensable, comme jadis en théorie, s'il veut mettre tous les atouts de son côté pour trouver un poste. Et la tendance sera probablement amplifiée par l'effet mécanique des recrutements. Mais il n'y a pas de raison de tirer la conclusion que bientôt on ne travaillera plus aux EtatsUnis que sur des écrivains vivants et/ou francophones (192-3).

L'interprétation de Compagnon qui semble faire de la francophonie un phénomène de mode important mais probablement passager et qui relèverait simplement de la «certification complémentaire » nous paraît problématique. Dominick LaCapra, à l'opposé, considère que les études françaises et francophones ne doivent pas se tourner le dos mais devraient au contraire se tourner les unes vers les autres puisqu'elles sont amenées à coexister :

The turn to the francophone should in fact mean a turning of both the francophone and the metropolitan towards one another in order to elicit their tangled relations, their often lost opportunities, and their possibilities for the future. [...] In any case, it would seem as unrealistic to propose a return to a purely canonical understanding of French literature as to postulate the viability of a purely francophone orientation. Problems raised by the

\footnotetext{
${ }^{6}$ Le terme «la francophonie » plus fréquemment utilisé au singulier ne fait pas justice à la diversité des cultures, littératures, traditions, histoires qui relèvent de l'aire francophone, diversité exprimée dans le néologisme Franco(poly)phonie. Nous observons dans cet article la distinction entre francophonie (avec minuscule) pour l'aire géographique sur laquelle le français est parlé ou existe en tant que langue officielle et Francophonie (avec majuscule) pour l'Organisation Internationale de la Francophonie (O.I.F.) créée en 1970 et qui regroupe actuellement 57 états membres.
}

This work is licensed under a Creative Commons Attribution 3.0 License 
objects of study and by their producers and readers would reveal the futility of such gestures (225).

La perception d'une compétition entre les disciplines résulte en partie de tensions qui existent aujourd'hui entre France et Francophonie qu'il importe de reconnaittre et d'interroger. La mise en relation «français-francophonie » n'est pas neutre mais souligne des tensions accrues depuis le début du vingt-etunième siècle, avec par exemple le débat controversé de 2009 sur l'identité nationale française. Lancé par le ministre de l'Immigration, de l'Intégration et de l'Identité Nationale, Éric Besson, le débat sur l'identité nationale de la France était censé encourager les Français à répondre à deux questions générales: «Pour vous, qu'est-ce qu'être français aujourd'hui? » et «Quel est l'apport de l'immigration à l'identité nationale?». Le débat, clos prématurément après trois mois de discussions qui tendaient vers le racisme et l'extrémisme, a cependant révélé que le français - langue, discipline, tradition, culture - se sent menacé aujourd'hui dans son hégémonie par les francophonies. Ces dernières, qui se sont de leur côté senties pendant longtemps marginalisées, "ghettoïsées» ou néo-colonisées par la francité rejettent dorénavant l'étiquette «francophone» afin de se resituer dans un contexte mondial plutôt que simplement hexagonal. Le manifeste littéraire publié dans Le Monde en 2007 intitulé «Pour une littérature-monde en français » annonçait ainsi la fin de la francophonie, devenue un concept trop encombrant et lourd de sens.

L'impact des études francophones sur les études françaises n'a cependant pas à être négatif. Les francophonies constituent une menace pour le français uniquement si ce dernier se cantonne dans une attitude passéiste, de «monumentalisation du français» (Cerquiglini 180) pour reprendre une expression de Bernard Cerquiglini, une monumentalisation qui ne pourrait être à la fois française et francophone alors qu'au contraire "Pluralisme bien ordonné commence par soi-même': le français est la raison de la Francophonie, le dialogue des langues, son ambition; la France, pays francophone, doit y adhérer avec ardeur» (Cerquiglini 2011: 185). Contre un impérialisme linguistique dépassé, il faut, selon une phrase souvent reprise de Michel Le Bris: « délier le pacte langue-nation, de sorte que la langue, libérée, devienne l'affaire de tous, en tous lieux» (Le Bris 46) ${ }^{7}$. Certaines instances gouvernementales sont a priori prêtes à accepter cette ouverture du français au

\footnotetext{
${ }^{7}$ Dans sa lecture critique du Manifeste des 44, Françoise Lionnet remarque à ce sujet que «The de-nationalization of literature has been proceeding apace for half a century if not more, but what is different now is that it has become official and very public, as a result of the manifesto, which is itself now the subject of articles, conferences, books, and special issues of journals that are thus causing much ink to flow » (209, italiques dans le texte).
} 
monde. Ainsi l'Ambassade de France aux États-Unis considère-t-elle la francophonie et sa «triple dimension » démographique, culturelle et éducative comme l'un des quatre atouts qui permettent au français de résister à l'érosion ${ }^{8}$. Les études francophones permettent de répondre à la question - si fréquemment posée - de l'utilité du français en ouvrant le monde et en soulignant que le français, langue moderne post-nationale, se parle également au Canada, en Belgique, en Suisse, au Sénégal, en Côte d'Ivoire, etc.

Souligner le rôle joué par les francophonies pour revitaliser le français court certes le risque de maintenir une approche néocolonisatrice contre laquelle Alain Mabanckou s'insurge à juste titre :

Au fond, dire d'un auteur francophone qu'il enrichit ou sauve la langue est loin d'être un compliment. De tels propos installent un lien de subordination: les lettres francophones ne sont vues que sous l'angle de leur utilité, de ce qu'elles apportent à la langue française (59, italiques dans le texte) $)^{9}$.

Les efforts d'assimilation ou d'exploitation de la francophonie pour améliorer la situation du français doivent être rejetés tout comme la tentation de faire fi du poids du passé doit être refoulée. Il importe, en effet, d'établir un constat des rapports complexes qui existent entre les deux disciplines afin de pouvoir les considérer comme deux systèmes qui facilitent les échanges et la réciprocité et éliminent les notions de centre et de périphérie dans ce que certains ont appelé la Francosphère. Comme le souligne Jean-Marc Moura dans Littératures francophones et théorie postcoloniale : "Les études postcoloniales conçoivent plutôt le français comme une langue au pluriel, dépourvue de centre évident » $(7)^{10}$. Un décentrement des études françaises - et non un remplacement -, et une ouverture qui inclut les études françaises parmi d'autres mettent en valeur la diversité des idiomes et des cultures face à la globalisation. L'alliance paraît en outre d'autant plus justifiée que les études françaises et francophones se trouvent chaque jour plus menacées par l'apparition des programmes d'études globales - "global studies» - dans les universités américaines qui risquent de conduire à l'élimination des particularismes remplacés par un nivellement culturel et linguistique.

\footnotetext{
${ }^{8}$ Les autres atouts sont : 1) «de bons chiffres » puisque le français est toujours la seconde langue enseignée aux Etats-Unis; 2) d'excellentes relations franco-américaines; 3) « un riche héritage de relations culturelles » (Service de coopération et d'action culturelle. Ambassade de France aux États-Unis 1075).

9 Mabanckou ajoute cette belle formule : «On n'écrit pas pour sauver une langue, mais justement pour en créer une [...]» (60).

10 Jean-Louis Joubert adopte la même position lorsqu'il affirme : «Il n’y a plus une littérature française, mais une polyphonie de voix littéraires qui enracinent la langue dans tous les continents » (cité par Moura 160).
}

This work is licensed under a Creative Commons Attribution 3.0 License 


\section{5) L'impact des études globales}

Le débat "études françaises versus francophonie(s)» qui remonte aux années $1990^{11}$ est en effet presque déjà un débat dépassé par l'apparition sur la scène des études globales qui non seulement posent différemment la question du rapport de la langue à la nation et à l'identité, mais soulèvent également la question de la survie de la langue et de la notion de nation dans un monde globalisant. Nous faisons ici une distinction importante entre d'une part la globalisation qui englobe, assimile les cultures et les langues et nivelle en ignorant les frontières et, d'autre part, la mondialisation qui rapproche, reconnaît et ouvre ces frontières en mettant les cultures en conversation; la première tombe dans le domaine des études globales et la seconde dans celui des études internationales.

Les études globales donnent certes accès aux études culturelles nationales faites en traduction - c'est-à-dire en anglais - et font, grâce à cette méthodologie, découvrir de nombreuses littératures-monde. De ce point de vue, on peut considérer avec Françoise Lionnet l'expansion des études globales comme un résultat positif de la globalisation du marché économique :

Over the past two decades, economic globalization has resulted in increased student interest in international studies, as well as in the global cultural realities that are the legacy of the colonial era. Identity, nation, and language have become prime topics of research in the humanities and social sciences, whether scholars are dealing with contemporary immigration issues or the history of nation formation (1252).

Lionnet se réjouit de l'augmentation des étudiants qui s’intéressent dorénavant aux autres cultures et espère « recruter» de futurs étudiants de français à partir de cette nouvelle vague d'étudiants en "réalités culturelles globales». On a depuis longtemps pu étudier la littérature française ou francophone en traduction et les départements d'anglais ou de littératures comparées dans les universités américaines enseignent régulièrement les œuvres du canon littéraire écrit en français, peut-être d'ailleurs davantage que les départements de français plus aptes à présenter des textes moins canoniques dans la langue originale. Cependant, une connaissance de la langue - principalement écrite et parfois rudimentaire - était jusqu'à présent toujours nécessaire dans ces départements, ce qui est de moins en moins le cas dans les programmes d'études globales qui tendent à la "glottophagie » pour reprendre un terme de Louis-Jean Calvet. Les programmes d'études globales se présentent comme des programmes hybrides dont le curriculum combine des classes de différents programmes, nouvelle version de ce que nous appelions autrefois les « doubles

\footnotetext{
11 Voir les deux numéros spéciaux de 1993 de Yale French Studies: «Post/Colonial Conditions: Exiles, Migrations, Nomadisms » édités par Françoise Lionnet et Ronnie Scharfman.
} 
spécialisations ", à ceci près que ces nouveaux programmes poussent vers les connaissances générales, y compris pour ce qui est des compétences linguistiques. Une spécialisation en français dans le cadre d'une licence de français ou d'une seconde spécialisation réclame généralement entre 32 et 36 heures de cours de langue, culture, etc.; tandis qu'une spécialisation en français dans le cadre d'une licence en études globales nécessite entre 12 et 15 heures. La différence constitue une menace à la fois pour le français en tant que discipline et en tant que langue ${ }^{12}$. L'intérêt pour tout ce qui relève des études globales est réel et peut motiver des étudiants à suivre nos classes de langue débutantes et intermédiaires; le risque cependant est cette fois-ci au niveau de la compétence linguistique. Le monde francophone parle français tandis que le monde global - ou le monde de la Littérature-Monde ou World Literature parle anglais de sorte que si « [...] encore une fois, la 'maladie' du français a un nom, celui d'une autre langue » (Calvet 249) : cette autre langue est l'anglais et non pas le «francophone ». Emily Apter exprime une préoccupation similaire vis-à-vis des programmes de World Literature lorsqu'elle remarque qu'il arrive trop souvent que :

[...] universities seize on World Literature as a catch-all rubric for flimsy programmes in the humanities that ignore rather than deepen local knowledge. World literature is all too conducive to the downsizing of 'foreign' language departments, and further monolingual coverage of literatures and area studies in other languages (288-89).

\section{6) Que faire?}

Confrontés à une situation complexe et changeante, les programmes de français ont peut-être tendance à apporter des changements rapides et ponctuels, mais souvent insuffisants afin de répondre aux messages alarmistes de crise du français et ainsi survivre aux attaques un semestre ou une année académique à la fois. Charles Forsdick mentionne par exemple la création d'un poste de professeur qui couvrirait l'ensemble de la francophonie : "This "becoming-postcolonial" of the field is not a matter of bolting on subsidiary material or of appointing what has been dubbed a "Francophone hire", a colleague who is expected to teach every non-metropolitan subject from New Caledonia to Haiti » (2011: 95).

De même le changement de nom des départements de langues que l'on remarque un peu partout ces dernières années - de "Foreign Languages and Literatures» à «World Languages» ou à "Languages, Cultures and International Trade » comme ce sera le cas à Southern Illinois University Carbondale à partir de l'automne 2014 - ne constitue qu'un pis-aller s'il ne s'accompagne pas d'un

\footnotetext{
12 Spécifions cependant que certaines universités comme l'université de l'Illinois à Urbana Champaign par exemple, exigent un semestre à l'étranger pour la licence en études globales.
}

This work is licensed under a $\underline{\text { Creative Commons Attribution 3.0 License }}$ 
remaniement en profondeur du curriculum et de l'orientation des départements. Il faut au contraire tirer avantage de la période actuelle de mutation pour réorienter les études françaises/ francophones/ francopolyphoniques/ francosphériques - il serait utile de s'entendre sur un terme! - et analyser la façon dont elles peuvent participer dans le monde globalisant non seulement afin de préparer des citoyens globaux qui puissent fonctionner dans une réalité complexe, mais également afin de créer un domaine d'études dans lequel les oppositions binaires - français/francophone, colonial/postcolonial, national/foreign - se trouvent remplacées par une ouverture au monde qui permette une « expansion of the national into a wider transnational sphere of enquiry » (Forsdick 2011: 99) ${ }^{13}$. Les études françaises doivent devenir postcoloniales et s'intégrer aux francophonies, elles doivent devenir transnationales et considérer les phénomènes de mouvement des populations, elles doivent encore devenir internationales et promouvoir le rôle $\mathrm{du}$ français en tant que moyen de communication entre pays. Les études françaises doivent se faire postcoloniales, transnationales et internationales non pas dans un réflexe de survie, mais dans un mouvement raisonné et constructif de renouveau qui leur offre une place comme domaine à part entière des études globales.

Les annonces fréquentes de la crise des études françaises, les tensions qui existent entre français et francophonie, le marketing des études plus «sexy» qui se vendent mieux à la fin du doctorat, l'explosion récente des études internationales et globales, la promotion de l'enseignement des langues cruciales au détriment des «vieilles langues européennes », tout cela participe à une image négative du français et néfaste au français en tant que langue et discipline. On regrette alors l'absence d'une structure, d'une organisation aux États-Unis - une association américaine des études francosphériques, par exemple - qui permettrait aux professeurs de se rencontrer afin précisément d'établir des lignes de conduite, un agenda à long terme, une orientation proposée à grande échelle pour faire face aux menaces d'une façon organisée plutôt qu'individuelle ou départementale. Il existe bien une Association Internationale des Etudes Françaises (AIEF) qui rassemble les professeurs de français, mais comme le relève Antoine Compagnon, «signe de leur indépendance post-coloniale, les Américains sont à peu près totalement

\footnotetext{
13 «In the light of such a concept, what concerns me is that the expansion of a purview of French studies to a wider Francosphere should not simply be seen as a response to a decline of the field, but should also encourage the development of a set of analytic practices that erode the residually binary relationship implied by the designation "French and Francophone", and permit France itself, and the French-speaking European zone of which it forms a part, also to be seen -through the study of phenomena as varied as regional language policy, national identity, and migrant literature - as globalised and postcolonial” (Forsdick 2010: 96).
} 
absents des instances et des congrès de l'AIEF » (185). Une telle structure permettrait de rapporter une certaine «discipline» dans l'indiscipline ${ }^{14}$ créée par l'interdisciplinarité et la pluridisciplinarité qui marquent depuis les années 1990 les études françaises, francophones et postcoloniales. En effet, tandis que Larry Norman fait l'éloge de l'indisciplinarité des études littéraires aux ÉtatsUnis $^{15}$, Charles Forsdick souligne :

The need to return to - or possibly even assert for the first time - a sense of disciplinariness of French studies, which may provide a clear foundation for the interdisciplinary dialogues in which we are invariably and inevitably engaged, but that will at the same time ensure that the enabling diversity by which our field has been characterized for several decades now does not tip into a disabling, disintegrating fragmentation (2010: 95).

Afin de s'opposer à cette fragmentation, il importe de développer une structure au niveau de la discipline des études francosphériques de sorte que les efforts des «défenseurs » de la cause du français ne restent pas isolés et insuffisants. De nombreuses organisations, conférences et sociétés diverses pour les études françaises maintiennent jusqu'à présent une répartition par siècle ou par spécialisations qui ne correspondent plus guère à l'enseignement $\mathrm{du}$ français et de moins en moins à la recherche des professeurs de français. Sans une approche méthodique, à long terme et généralisée de l'orientation des études de la francosphère et de leur signification dans un monde globalisant, les décisions ponctuelles risquent fort d'être prises pour nous, souvent par des administrateurs non conscients des enjeux.

\footnotetext{
${ }^{14}$ L'expression est de Larry Norman: «Car il me semble que les études littéraires en Amérique ont longtemps eu pour marque distinctive de ne pas s'organiser en domaines de compétences clairement identifiés et qu'il est temps de faire l'éloge moins de l'interdisciplinarité que de l'indisciplinarité, ou tout du moins d'une approche qui allie à une méthodologie suffisamment rigoureuse une lecture critique qui sache s'écarter de la discipline ». (Norman 22).

15 «Le grand atout de cette formation indisciplinée (ou, si l'on veut, disciplinée sans disciplinarité) est que notre étudiant, libre de toute idée rigoureusement disciplinaire, est aussi libre de toute idée reçue et échappe ainsi aux poncifs qui gouvernent, qui disciplinent, trop souvent nos discours ». (Norman 32-33, italiques dans le texte).
}

This work is licensed under a Creative Commons Attribution 3.0 License 


\section{Bibliographie}

Apter, Emily. "Afterword: The 'World' in World Literature.” Hargreaves, Alec, Charles Forsdick, and David Murphy, eds. Transnational French Studies. Postcolonialism and Littérature-monde. Liverpool: Liverpool University Press, 2010. 287-295.

Ben Jelloun, Tahar. "La cave de ma mémoire, le toit de ma maison sont des mots français." Pour une littérature-monde en français. Michel Le Bris et Jean Rouaud, eds. Paris: Gallimard, 2007. 113-124.

Calvet, Louis-Jean. La guerre des langues et les politiques linguistiques. Paris: Payot, 1987.

Calvet, Louis-Jean. “The 'Weight' and Prognosis of Languages: a Theory Applied to French." La Francophonie ou l'éloge de la diversité. Michael Abecassis, Gudrun Ledegen et Karen Zouaoui, eds. Newcastle upon Tyne, UK Cambridge Scholars Publishing, 2011. 129-150.

Cerquiglini, Bernard. "Les rendez-vous manqués du pluralisme français." La Francophonie on l'éloge de la diversité. Michaël Abecassis, Gudrun Ledegen, and Karen Zouaoui, eds. Cambridge Scholars Publishing, 2011. 179185.

Compagnon, Antoine. "Intervention d'Antoine Compagnon : Les États-Unis," La traversée des thèses. Bilan de la recherche doctorale en littérature française du XXe siècle. Actes du colloque de la Société d'Étude de la Littérature Française du XXe siècle (SELF XX). Eds. Didier Alexandre, Michel Collot, Jean-Yves Guérin, Michel Murat. Paris: Presses Sorbonne Nouvelle, 2003. 185-193.

Forsdick, Charles. "“On the Abolition of the French Department'? Exploring the Disciplinary Contexts of Littérature-monde." Hargreaves, Alec, Charles Forsdick, and David Murphy, eds. Transnational French Studies. Postcolonialism and Littérature-monde. Liverpool: Liverpool University Press, 2010. 89-108.

Forsdick, Charles. "Mobilising French Studies." Australian Journal of French Studies 48.1 (2011): 88-103.

Furman, Nelly, David Goldberg and Natalia Lusin. "Enrollments in Languages Other than English in United States Institutions of Higher Education, Fall 2009." www.mla.org/pdf/2009 enrollment survey.pdf. Web publication, décembre 2010. 1-41.

Hargreaves, Alec, Charles Forsdick, and David Murphy. Transnational French Studies. Postcolonialism and Littérature-monde. Liverpool: Liverpool University Press, 2010.

LaCapra, Dominick. History and Reading. Tocqueville, Foucault, French Studies. Toronto: University of Toronto Press, 2000. 
Le Bris, Michel. "Pour une littérature-monde en français." Pour une littératuremonde. Michel Le Bris et Jean Rouaud, eds. Paris: Gallimard, 2007. 2353.

Lionnet, Françoise. "National Language Departments in the Era of Transnational Studies." PMLA 117.5 (2002): 1252-1254.

"Universalisms and Francophonies." International Journal of Francophone Studies 12.2-3 (2009): 203-21.

Mabanckou, Alain. "Le chant de l'oiseau migrateur." Pour une littérature-monde en français. Michel Le Bris et Jean Rouaud, eds. Paris: Gallimard, 2007. 5566.

Moura, Jean-Marc. Littératures francophones et théorie postcoloniale. Paris: Presses Universitaires de France, 1999.

Moudileno, Lydie. "Francophonie: Trash or Recycle?" Hargreaves, Alec, Charles Forsdick, and David Murphy, eds. Transnational French Studies. Postcolonialism and Littérature-monde. Liverpool: Liverpool University Press, 2010. 109-24.

Norman, Larry. "La transformation des disciplines dans l'université américaine." Cabiers de l'Association Internationale des Études Françaises 58.58 (2006): 21-34.

Service de coopération et d'action culturelle. Ambassade de France aux ÉtatsUnis. "Le français aux États-Unis: une priorité de l'Ambassade." The French Review 86.6 (2013): 1072-93.

Victor, Gary. "Littérature-monde ou liberté d'être." Pour une littérature-monde en français. Michel Le Bris et Jean Rouaud, eds. Paris: Gallimard, 2007. 315-20.

This work is licensed under a Creative Commons Attribution 3.0 License 